Review

\title{
Sensorimotor cortex as a critical component of an 'extended' mirror neuron system: Does it solve the development, correspondence, and control problems in mirroring?
}

\section{Jaime A Pineda}

Address: Departments of Cognitive Science and Neuroscience, University of California, San Diego, La Jolla, CA 92037-0515, USA

Email: Jaime A Pineda - pineda@cogsci.ucsd.edu

Published: 18 October 2008

Behavioral and Brain Functions 2008, 4:47 doi:10.1186/1744-9081-4-47
Received: 10 June 2008

Accepted: 18 October 2008

This article is available from: http://www.behavioralandbrainfunctions.com/content/4/1/47

(c) 2008 Pineda; licensee BioMed Central Ltd.

This is an Open Access article distributed under the terms of the Creative Commons Attribution License (http://creativecommons.org/licenses/by/2.0), which permits unrestricted use, distribution, and reproduction in any medium, provided the original work is properly cited.

\begin{abstract}
A core assumption of how humans understand and infer the intentions and beliefs of others is the existence of a functional self-other distinction. At least two neural systems have been proposed to manage such a critical distinction. One system, part of the classic motor system, is specialized for the preparation and execution of motor actions that are self realized and voluntary, while the other appears primarily involved in capturing and understanding the actions of non-self or others. The latter system, of which the mirror neuron system is part, is the canonical action 'resonance' system in the brain that has evolved to share many of the same circuits involved in motor control. Mirroring or 'shared circuit systems' are assumed to be involved in resonating, imitating, and/or simulating the actions of others. A number of researchers have proposed that shared representations of motor actions may form a foundational cornerstone for higher order social processes, such as motor learning, action understanding, imitation, perspective taking, understanding facial emotions, and empathy. However, mirroring systems that evolve from the classic motor system present at least three problems: a development, a correspondence, and a control problem. Developmentally, the question is how does a mirroring system arise? How do humans acquire the ability to simulate through mapping observed onto executed actions? Are mirror neurons innate and therefore genetically programmed? To what extent is learning necessary? In terms of the correspondence problem, the question is how does the observer agent know what the observed agent's resonance activation pattern is? How does the matching of motor activation patterns occur? Finally, in terms of the control problem, the issue is how to efficiently control a mirroring system when it is turned on automatically through observation? Or, as others have stated the problem more succinctly: "Why don't we imitate all the time?" In this review, we argue from an anatomical, physiological, modeling, and functional perspectives that a critical component of the human mirror neuron system is sensorimotor cortex. Not only are sensorimotor transformations necessary for computing the patterns of muscle activation and kinematics during action observation but they provide potential answers to the development, correspondence and control problems.
\end{abstract}




\section{Background}

Human beings are social creatures to the extent that interactions with members of their own species, and especially the ability to understand and infer the intentions and beliefs of others, has become of predominant importance in their daily life. Whether for cooperation or non-cooperation, a core assumption of this viewpoint is that such social interactions spring from a distinction between self and others. It can be argued that at least two hierarchically-organized, overlapping and interacting neural systems have evolved and developed to manage self-other distinctions and hence social interactions [1]. One system, part of the classic motor system, is more specialized for the preparation and execution of motor actions that are self realized and voluntary, while the other appears to be more involved in capturing and understanding, at a basic and involuntary level, the actions of non-self or others. For our purposes, actions are defined as sequences of movements that together solve a motor problem [2] and that involve at least four levels of behavioral complexity: intention, kinematics, goal-object identity, and the physical consequences of the action [1]. Motor preparation and execution circuitry includes, among others, the premotor cortex, supplementary motor area, sensorimotor cortices, and parts of the inferior parietal cortex. The second system, of which the mirror neuron system (MNS) is part, has been described as the canonical action 'resonance' system in the brain - one that has evolved to utilize or share many of the same circuits involved in motor control [3]. Mirroring or 'shared circuit' systems are assumed to be important for resonating, imitating, and/or simulating the actions of others. Although no consensus exists, a number of researchers have proposed that shared representations of motor actions, or the action understanding properties of this system, may form a foundational cornerstone for higher order social processes, including motor learning, action understanding, imitation, perspective taking, understanding facial emotions, and empathy [4-8]. This means that adopting someone else's viewpoint or perspective at the very least requires that the other's actions be understood; else no accurate prediction of their behavior can be made.

However, a mirroring system that evolves and is adapted from the classic motor system presents at least three major problems: a development, a correspondence, and a control problem. In terms of the development problem the question is whether humans acquire the ability to mirror by mapping observed onto executed actions? That is, how exactly does a mirroring system arise? Are mirror neurons innate and therefore genetically programmed? Is learning necessary? And, what role does sensorimotor cortex play? A number of studies have indicated that imitation of facial and hand gestures in both human and non-human primates suggest the existence of mirroring systems in infancy [9-11]. Likewise, electroencephalography (EEG) and near infrared spectroscopy studies in humans show sensitivity to executed versus observed actions, as well as between live and televised actions [12-16] suggesting the existence of mirroring as early as 6-7 months of life. However, none of these studies directly answers the development questions posed. On the other hand, computational models of mirroring activity propose that sensorimotor transformations, via Hebbian learning, can in fact mediate such development.

In terms of the correspondence problem the question is how does the observer agent determine what the observed agent's activation pattern is in order to match it? Or, as Brass and Heyes [17] stated the problem with respect to imitation, "When we observe another person moving we do not see the muscle activation underlying their movement but rather the external consequences of that activation. So, how does the observer's motor system 'know' which muscle activations will lead to the observed movement?" Resonance becomes particularly difficult when the observer and observed do not share the same embodiment and affordances, that is, they do not share all "action possibilities" latent in the environment. One partial solution to this problem, of course, exists in the implicit nature of a mirroring system, i.e., a system that evokes motor representations by movement observation. That is, if motor actions already exist as part of the observer agent's movement repertoire then observation of action, even when partially triggered, can be sufficient to evoke the representation. This solution clearly makes sensorimotor transformations, as part of a mirroring system, necessary for solving such a correspondence problem.

Finally, in terms of the control problem, the issue arises because an efficient mirroring system ought to be turned on only when needed. However, it has been shown repeatedly that activation of internal motor representations via observation occurs automatically. Neuroimaging studies, for example, show that simple passive observation is enough to generate motor activation. The question then is how to control a system for efficiency when it is turned on automatically? Or, as others have stated the problem succinctly: "Why don't we imitate all the time?" The existence of neural inhibitory and monitoring mechanisms as partial solutions to this control problem has been acknowledged [3], although the specific anatomical implementation of such mechanisms is unknown. Brass and colleagues [18], for example, found that the frontomedian cortex and the right temporo-parietal junction were activated when an instructed movement had to be executed during observation of an incongruent movement. The implication being that high level areas are involved in inhibition of imitative response tendencies. Another solution centers on phasic changes in oscillatory 
EEG activity as inhibitory control mechanisms. This is consistent with the role of sensorimotor cortex as a critical region for mirroring based on its common output path role in motor and simulation-based representations. More specifically, we hypothesize that oscillatory activity, such as mu rhythms in sensorimotor cortex, play a key role in controlling mirroring processes.

Mirroring activity can be conceptualized as occurring in a gradient. At one end of the spectrum, the mimicry of another individual's postures, facial expressions, vocalizations, movements and mannerisms is often executed in the absence of awareness, as occurs in the chameleon effect, motor empathy, motor contagion, or emotional contagion [19]. At the other end of the spectrum, it has been suggested that simulation based on mapping of observed actions onto one's own motor system necessitates the interaction with semantic/cognitive circuits for conscious action understanding to occur $[20,21]$. We conceptualize this spectrum of action understanding as reflecting four levels of behavioral complexity, i.e., intentions, goals, patterns of muscle activation, and kinematics, as has been suggested by Hamilton and Grafton [1]. Furthermore, we argue that these levels of processing can be mapped onto differences in activation in different components within a 'core' and an 'extended' mirror neu- ron system (see Figure 1). Although it remains to be definitively shown, differential activation of the various components of this mirroring system most likely result as a function of the task, working memory, motivational and/or attentional factors involved. In this paper, we argue from an anatomical, physiological, modeling, and functional perspectives that one critical component of an 'extended' mirror neuron system is sensorimotor cortex. This region is necessary not only for computing the patterns of muscle activation and kinematics during action observation but provides potential answers to the development, correspondence and control problems in mirroring.

\section{The 'core' MNS}

The mirror neuron system has been widely defined as consisting of three interrelated areas: ventral premotor area (PMv) of the inferior frontal gyrus (area F5 in monkeys), parietal frontal (PF) in the rostral cortical convexity of the inferior parietal lobule (IPL), and the superior temporal sulcus (STS) (see Figures 1, 2 and 3, as well as Table 1 for a description of these areas). The mirror neuron circuit in monkeys [4,22] begins in the rostral part of the superior temporal sulcus, although no mirror neurons per se have been reported in this area. Information is then thought to flow to the parietal frontal area on the rostral cortical con-

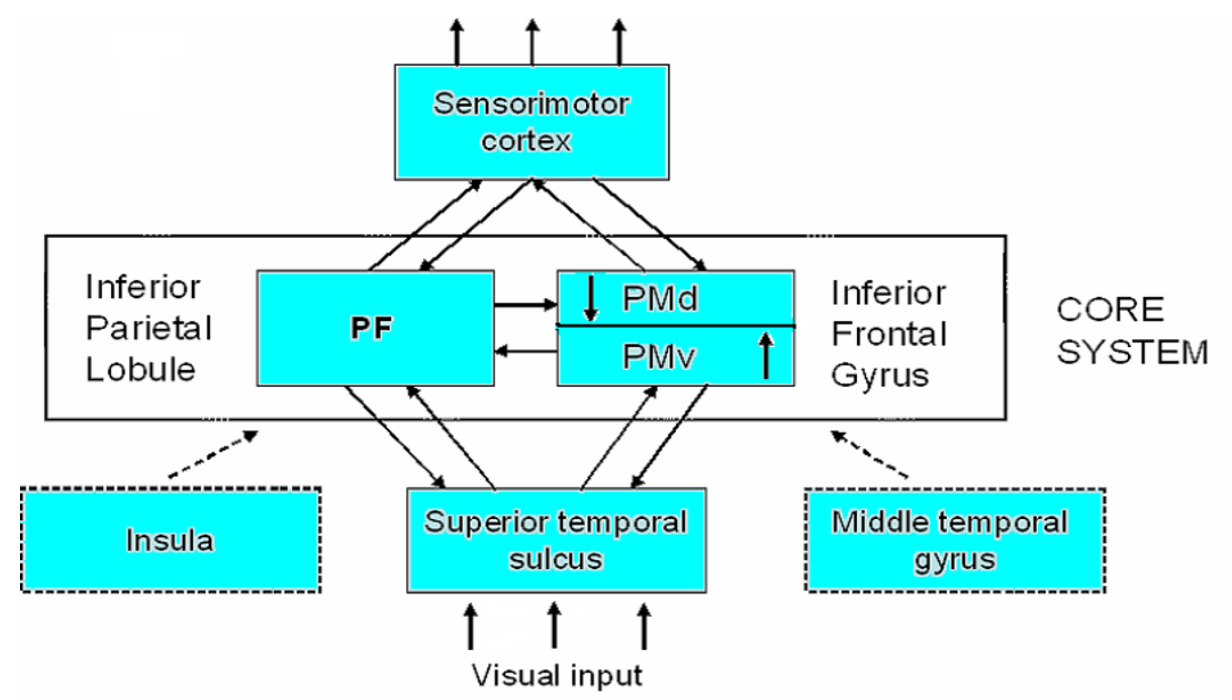

EXTENDED MIRROR NEURON SYSTEM

Figure I

Schematic of areas in the human brain that contain mirror neurons (inferior parietal lobule and inferior frontal gyrus) and make up the 'core'system. The 'extended' mirror neuron system involves additional brain areas, e.g., insula, middle temporal gyrus, and somatosensory cortex, which connect to the core system and perform transformations on the data critical for mirroring and simulation. 
vexity of the inferior parietal lobule. A subset of the cells in this region has mirror properties: i.e., they discharge both when the monkey executes as well as observes an action. Parietal frontal area, in turn, sends projections to area F5 of the ventral premotor area, where a subset of cells (10-20\%) exhibits mirror properties. Thus, the core mirror neuron system would be defined as those areas that contain mirror-like neurons, which at this point includes primarily the rostral convexity of the inferior parietal lobule or parietal frontal area and ventral premotor area.

Single unit studies in the premotor cortex of macaque monkeys indicate that neurons in area F5, particularly in the caudal portion of the inferior frontal gyrus (IFG), are indistinguishable from neighboring neurons in terms of their motor properties and discharge in response to executed and observed actions [23] (for a review see [4]). The implication is that when a monkey observes an action, particularly one that is in its motor repertoire, a subset of neurons in this region 'mirrors' the activity and represents the motor action in its own premotor cortex, revealing a type of observation/execution matching system. This type of observation/execution activity has been shown to be selective for goal-directed, meaningful actions supporting the idea that actions are organized with respect to distal goals [24]. More recently, another subpopulation of neurons in the same area of the monkey has been found that discharges both when the animal performs a specific action as well as when it sees or hears the same action performed by another individual $[25,26]$. That is, these cells represent in an individual's motor cortex not only the execution of an action (motor representation) but also the 'observation' of that action performed by others (visual representation), as well as its auditory correlates (auditory representation). In other words, auditory mirror neurons allow for a mapping of specific heard actions onto the motor programs for executing the same actions.

Individual human mirror neurons cannot be studied directly except under unusual circumstances [27]. Nonetheless, the evidence suggests that the motor related part of Broca's region is located in the caudal portion of the inferior frontal cortex, in what is Brodmann's area 44, and there appears to be a homology between area F5 in the monkey and area 44 in humans. Area 44 is involved in interfacing external information about biological motion and internal motor representation of hand/arm and mouth actions $[28,29]$. Hence, the existence of an analogous mirroring system in the homologous human brain regions has been supported by indirect population-level measures such as electroencephalography [12,30-34], magnetoencephalography [35], transcranial magnetic stimulation [36], positron emission tomography $[37,38]$ and functional magnetic resonance imaging $[19,39,40]$.
Fadiga and colleagues [36], for example, found that motor evoked potentials over motor cortex were enhanced in response to transcranial magnetic stimulation when subjects observed another individual performing an action relative to when they detected the dimming of a light. Iacoboni and colleagues [39] measured blood flow in Brodmann's area 44 and found increases during the observation and performance of actions. Other studies have reported activations with similar properties in the parietal cortex $[40,41]$, as well as the superior temporal sulcus $[42,43]$. In general, the human mirror neuron system appears active during the performance and observation of the same action and is hypothesized to be necessary for imitative learning [44], comprehending the actions of others [24,45], understanding the goal of another's actions [46], interpreting facial expressions [19,47], and exhibiting empathy [19].

\section{The 'extended' MNS}

It has been shown that we activate our own motor, somatosensory, and nociceptive representations while perceiving the actions of others, while at the same time activating representations of our own emotional states as well as facial expressions while witnessing others' emotions [48]. At minimum, this activation of shared representations for action and emotion requires a variety of anatomical and functional circuits that together might be called the 'extended' mirror neuron system. Undoubtedly, the core mirror neuron areas, as described previously (see Figure 1 ), are anatomically connected with many other regions that contribute significantly to the subsequent elaboration of the information $[49,50]$. Those regions may themselves not contain mirror neurons per se, such as the superior temporal sulcus, but the level of transformation performed on the data would make them critical to the outcome and part of an extended mirroring process.

The arguments as to why the superior temporal sulcus, despite the lack of mirror neurons, is considered part of a mirror neuron system are both anatomical and functional [51]. It is an area that contains neurons that respond to biologically relevant actions of the head, body, and eyes, as well as to static pictures that merely imply biological motion [52]. Furthermore, this area is reciprocally connected to the parietal frontal area in the inferior parietal lobule. However, the functional significance of the mirror neuron system has to be understood in its connections to many other neural systems [20]. Thus, the degree to which brain areas in these other systems play a critical role in action understanding or in any of the processes attributed to the core mirror neuron system would define their inclusion as part of an extended circuit.

The extant evidence supports inclusion of a number of areas into an extended definition of the mirror neuron 

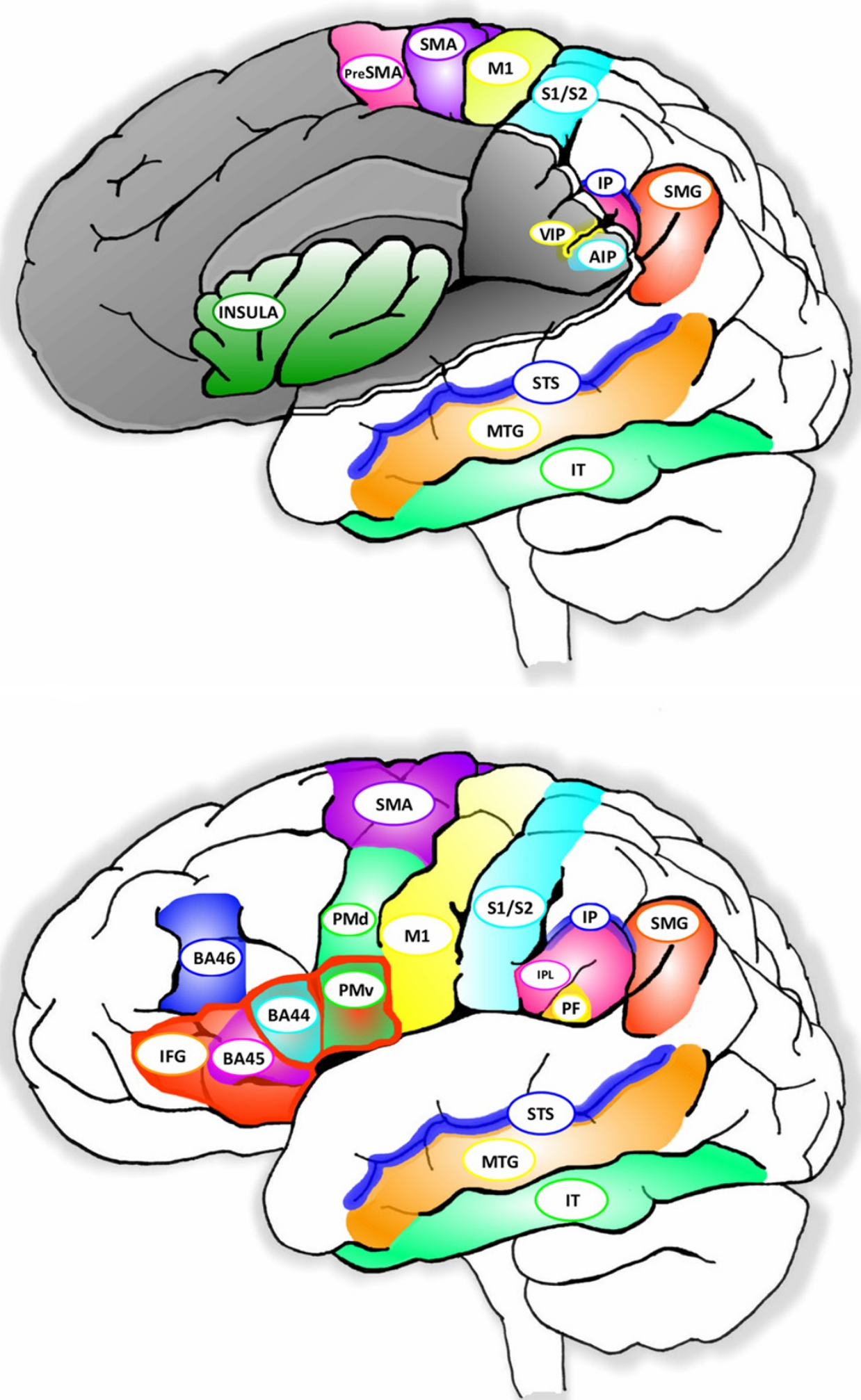

Figure 2

Anatomical view of a human brain showing areas involved with the mirror neuron system. 

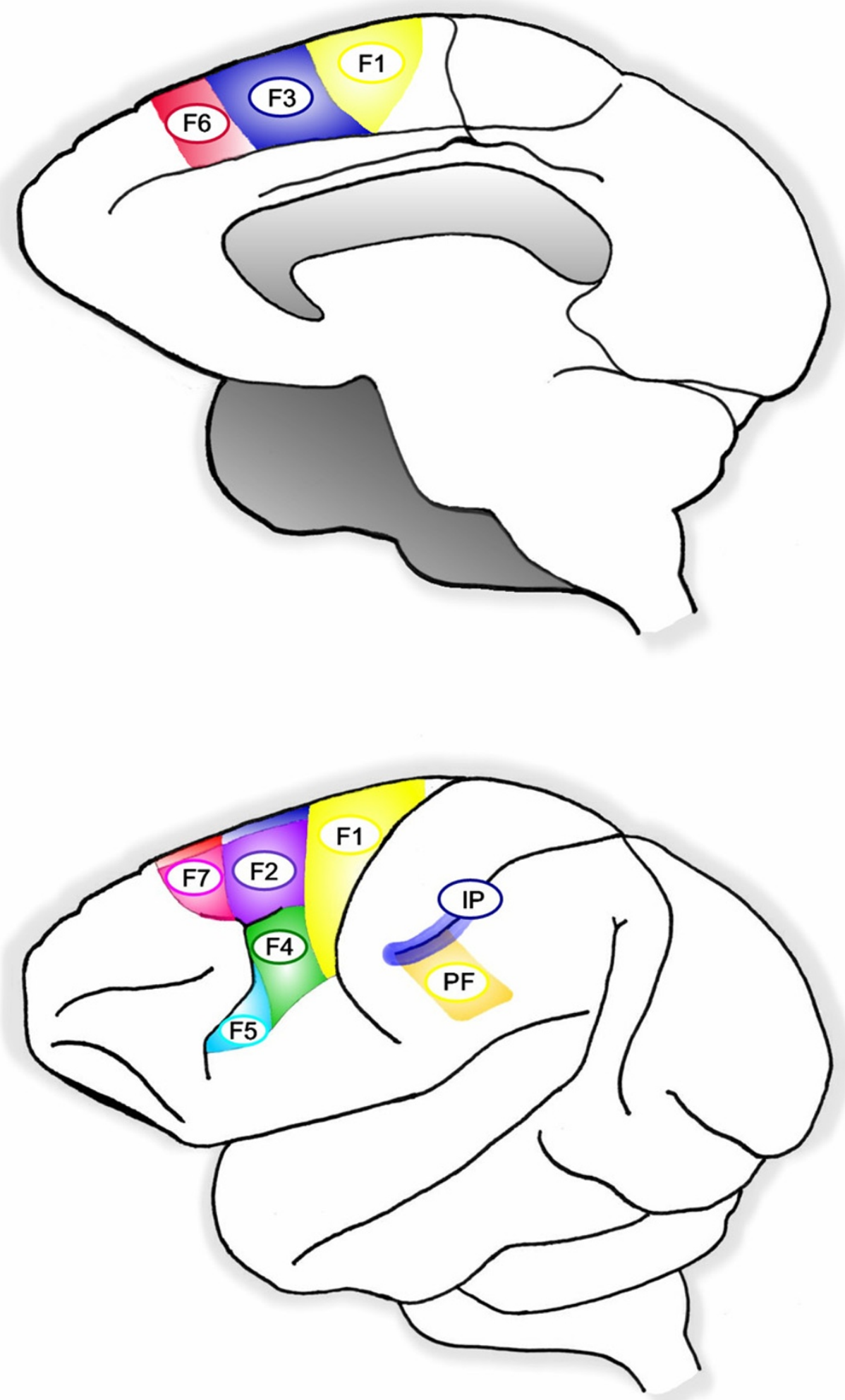

Figure 3

Anatomical view of a macaque monkey brain showing areas involved with the mirror neuron system. 
Table I: Abbreviations and functional descriptions of anatomical areas

\begin{tabular}{|c|c|c|}
\hline Abbreviation & Name & Function \\
\hline AIP & Anterior intraparietal & visually guided grasping; comparable to monkey area F5 \\
\hline BA44 & Brodmann's area 44 & Broca's area; language production \\
\hline BA46 & Brodmann's area 46 & rostral portion of the IFG; sustained attention and working memory \\
\hline $\mathrm{F} 2$ & Monkey area F2 & integrates body position and motor acts \\
\hline bF4 & Monkey area F4 & codes for peripersonal space; caudal part of PMv \\
\hline F5 & Monkey area F5 & codes for distal movements; rostral part of PMv \\
\hline F6 & Monkey area F6 & pre-SMA; learning of new motor sequences \\
\hline IFG & Inferior frontal gyrus & action observation and imitation \\
\hline Insula & Insular cortex & body representation and subjective emotional experience \\
\hline IP & Intraparietal sulcus & guidance of limb and eye movement \\
\hline IPL & Inferior parietal lobule & $\begin{array}{l}\text { post-central sulcus/anterior border, intraparietal sulcus/superior border, and the lateral fissure/ } \\
\text { anterior inferior border. }\end{array}$ \\
\hline IT & Inferotemporal cortex & identification and categorization of objects \\
\hline MI & Primary motor cortex & patterns of muscle activation \\
\hline MTG & Middle temporal gyrus & $\begin{array}{l}\text { subserves language and semantic memory processing, visual perception, and multimodal sensory } \\
\text { integration }\end{array}$ \\
\hline PF & Parietal frontal & rostral convexity of IPL \\
\hline PMd & Dorsal premotor & simultaneous encoding of multiple movement \\
\hline PMv & Ventral premotor & monkey area F5; analogous to BA 44; pars opercularis of IFG \\
\hline SI & Primary somatosensory & kinematics \\
\hline S2 & Secondary somatosensory & integrating across body parts; frontoparietal operculum and lateral convexity of IPL \\
\hline SMA & Supplementary motor & planning motor actions \\
\hline SMG & Supramarginal gyrus & spatial orientation and semantic representation \\
\hline STS & Superior temporal sulcus & visual information entry area \\
\hline VIP & Ventral intraparietal & comparable to monkey area F4 \\
\hline
\end{tabular}

system. For example, the subjective sense of how one feels is theorized to be based upon anterior insula representations of the body. This is assumed to provide a foundation for emotions and perhaps even for self-awareness that could allow for simulation of future actions, in order to use the feelings generated by the simulation to guide decision making [53]. Singer and colleagues [54] found, in a functional magnetic resonance imaging study, that empathy for pain involves simulating the unpleasant, aversive qualities of the pain (the motivational significance of 
pain) but not its precise somatic characteristics. In another study, Saarela and Hari [55] used photos of facial expressions from chronic pain sufferers which varied in the intensity of depicted suffering. Not only were bilateral anterior insula, left anterior cingulate, and left inferior parietal lobe activated, but the amount of these activations correlated with subjects' estimates of the intensity of observed pain. Clearly, the insula has an important role in mirroring and should be considered part of the extended mirroring system. Likewise, observation-evoked motor activity, as well as mirror-type activity, has been reported in dorsal premotor cortices [56,57], while the middle temporal gyrus (MTG) and adjacent superior temporal sulcus are often found to show augmented blood-oxygen level dependent (BOLD) responses during action execution and action observation $[58,59]$. Finally, and most relevant to the argument in this paper, primary and secondary motor and somatosensory cortices often contain voxels active during both action execution and observation/listening $[13,58,60,61]$.

\section{Anatomical perspective}

Sensorimotor cortex has been implicated in determining the organization and representation of conceptual knowledge of concrete objects and actions $[47,62,63]$. Behavioral and functional magnetic resonance imaging studies support the notion of mental representations grounded in sensorimotor interactions with the real world [64,65]. Such representations are most likely involved in understanding and producing actions and emotions of conspecifics via simulation of observed behavior $[24,47,66,67]$. In order to understand the role of sensorimotor cortex in mirroring, simulation and in understanding the actions of others, as well as to understand how sensorimotor cortex solves the development, correspondence and control problems, it is helpful to understand its anatomical and functional properties, and more precisely the underlying computations necessary for movement and movement understanding.

\section{MI connections}

A number of neurophysiological [13,68-70] and neuroimaging [71-73] studies have shown that mirror-like activity occurs in several brain regions including the human primary motor (M1) and somatosensory (S1) cortices. Why should these sensorimotor cortices be active during action observation? Since the majority of studies have examined hand movements, sensorimotor activation may simply be a side effect of the strong reciprocal connections between premotor cortex and sensorimotor areas. Premotor cortex is typically subdivided into dorsal (PMd) and ventral (PMv) regions (see Figure 2). Stepniewska et al. [74,75], Greenlee et al., [76] and Dum and Strick [77] have shown that the densest inputs from premotor areas to the orofacial and digit representation in primary motor cortex originate from dorsal and ventral premotor areas. The ventral premotor area connects with the digit and orofacial portions of primary motor cortex and also has extensive connections with somatosensory areas (S1, S2, 3a). Dorsal premotor area also connects with proximal forelimb and trunk areas of primary motor cortex [75] and is connected directly to spinal cord [78].

Dum and Strick [77] performed tracer studies in the Cebus monkey and employed a number of techniques, including electrophysiologically mapping the digit representations, to check against their tracer results, and used dual tracers to compare multiple inputs to the primary motor cortex in the same animal. The results showed that for digit representations, primary motor cortex receives the strongest input from the ventral and dorsal premotor areas. These areas in turn receive their strongest reciprocal input from primary motor cortex, and it appears that the same area in motor cortex projects to both ventral and dorsal premotor areas. Furthermore, there is also a strong amount of interconnection between the ventral and dorsal premotor areas as well. The argument made by Dum and Strick [77] is that such areas form a densely interconnected network concerned with the generation and control of hand movements. Hence, primary motor cortex is active because premotor areas are active. However, Kilner and Frith [51] offer an alternative explanation to this passive response activation. They suggest that premotor and primary motor areas code executed action in different coordinate systems. Premotor areas code targeted action primarily in an extrinsic reference framework that encodes the kinematic aspects of the action, that is, target and hand are defined relative to each other in space. In contrast, primary motor neurons code the same action based on an intrinsic framework of muscles and joint space that is related to the shaping of hand and digits. Therefore, understanding actions and inferring intentions require both the premotor areas for a kinematic description and primary motor cortex for a description of the patterns of muscle activity necessary to execute the action.

In a recent study examining single-cell properties of primary motor cortex and dorsal premotor area neurons, Tkach et al. [79] identified a set of cells that exhibited observation- and execution-based activation, a major characteristic of mirror neurons. However, their study did not show whether these cells also responded to the interaction between subject and target object, a characteristic of mirror neurons. In another study, Stefan et al. [80] showed that primary motor cortex displays mirror-like activity in response to movement observation, is capable of forming motor memories, and is involved in motor learning. In their study, transcranial magnetic stimulation was used to show that observation of another individual performing simple repetitive thumb movements gives rise 
to a kinematically specific memory trace of the observed motions in this motor region.

\section{SI connections}

In one of the first functional magnetic resonance imaging studies to examine 'tactile empathy,' Keysers et al. [81] showed that secondary somatosensory area (S2), in the fronto-parietal operculum, extending onto the lateral convexity of the inferior parietal lobule and presumably involved in integrating information across body parts, is activated both when the participants were touched and when they observed someone or something else getting touched by objects. This area receives somatosensory, visual, and polysensory inputs from primary somatosensory cortex, and extrastriate visual areas, as well as from areas in the posterior parietal lobe, suggesting that it may be involved in integrating somatosensory information with other sensory modalities [82]. Furthermore, this secondary somatosensory area has extensive reciprocal connections with ventral premotor areas, as well as with prefrontal cortex (Brodmann's area 46). Curiously, Keysers et al. [81] did not show primary somatosensory cortex activation to the observation of touch. In contrast, in a more recent study [83], it was reported that the primary somatosensory cortex was indeed activated in non-synesthesia subjects by the mere observation of touch and that this activation was somatotopically organized. Furthermore, the mirror neuron system in these subjects (including the premotor cortex, superior temporal sulcus, and parietal cortex) was activated by the observation of touch to another human more than to an object. Interestingly, in a synesthesia subject these areas appeared to be overactive, i.e., above the threshold for conscious tactile perception.

It has also been suggested that somatosensory representations are critical for processing emotion [84]. Adolphs et al. [85] provided a theoretical framework in which they suggested that recognizing emotion in another person engages both visual representations of the perceptual properties of facial expressions and somatosensory representations of the emotion that may simulate how one would feel if making the shown facial expression. Specifically, they found that lesions in the right somatosensory cortex, as well as in anterior supramarginal gyrus and to a lesser extent in the insula, were associated with impaired recognition of emotions from human facial expressions. Individuals having only somatosensory lesions showed impairment. They also reported a significant correlation between impaired somatic sensation and impaired recognition, but only in the right hemisphere and not shown in relation to motor impairments. A study by Hagen et al. [86] showed that posterior inferior frontal gyrus receives extensive projections from secondary somatosensory areas and responds to somatosensory stimulation. Infe- rior frontal gyrus also has projections from primary somatosensory cortex, area $7 \mathrm{~b}$, and the ventral frontal opercular region [82]. Monkey studies have suggested that functionally this region may be important for working memory for tactile stimuli [87].

\section{Computational perspective}

One computational view of mirror neuron functionality places it in the context of auto-associative networks whose links are strengthened via Hebbian synaptic plasticity. In this view, neurons become capable of sharing representations primarily through an associative learning mechanism. That is, these auto-associative or content addressable memory architectures are established when an agent acts. That is, associations naturally occur among the motor, somatosensory, vestibular, auditory, visual, and other inputs when a movement is executed. It is hypothesized that linking the observation of movement (visual input) to extant motor representations such that later observed actions can retrieve these stored patterns automatically can explain how the mirror neuron system develops. This notion of associational learning is supported by recent evidence showing that it is possible to manipulate the selectivity of the human mirror system, and thereby make it operate as a countermirror system, by giving participants training to perform one action while observing another [88]. These results by Catmur and colleagues strongly argue that mirroring is not entirely innate [9] nor unchangeable once the patterns are learned; but most likely develop through sensorimotor associational learning $[89,90]$ as a product and a process of social interaction.

This idea is also supported by neuroimaging studies that purport to show that mirror neuron activity varies as a function of the observer's expertise. Calvo-Merino et al. [91] showed that ballet and capoeira dancers observing actions they were trained to perform showed greater activity in premotor and parietal areas. Similarly, Haslinger et al. [92] showed similar effects for piano players observing piano playing. It's also been shown that familiarity (which presumably involves enhanced sensorimotor activation) activates premotor cortex more than non-familiar actions [33].

Oztop and Arbib [49,50] have argued that mirroring properties are an exaptation of a more basic neuronal function, namely that of providing feedback for visuallyguided grasping movements. Although the evolution of how such self-hand movements relate to objects to recognize the manual action of others is unclear, Fagg and Arbib [93] have suggested, based on various computational models, that dorsal stream information flowing through the anterior intraparietal area is where the grasps afforded by the object (i.e., those actions that are made 
possible by the object) are extracted, while area F5 selects and drives the execution of the grasp. Prefrontal cortex, which receives object recognition information from inferotemporal cortex (IT), biases F5 selection to choose the appropriate possible actions for the task. Furthermore, a variety of prefrontal areas, such as F6 (pre-SMA), Brodmann's area 46 (dorsolateral prefrontal cortex), and F2 (dorsal premotor cortex) are proposed to be involved in biasing F5 to respond to task constraints, working memory, and instruction stimuli, respectively. Once the location of the object is known, the information flows to the motor programming area $\mathrm{F} 4$, which computes the reach. The information about the reach and the grasp is fed into primary motor cortex to control the hand and arm.

Although this computational framework of how actions are organized with respect to distal goals is incomplete, there is agreement that primary motor cortex computes muscle activations given reach targets and limb postures in the presence of noise $[94,95]$. Other computational perspectives argue that mirroring systems involved in recognizing actions can be understood within a predictive coding framework, or more formally, as equivalent to Bayesian inference within a hierarchical structure [96]. This refers to a computational framework for inferring the causes (intentions, goals, and motor commands) of sensory inputs (observed kinematics) by minimizing prediction error at all levels of a cortical hierarchy. Indeed, the notion that muscle activity is a linear projection of primary motor cortex output has been called into question [97-99]. Rather, primary motor cortex receives input from ventral premotor area, which appears to code object locations in a hand-centered frame of reference. It then sends its output to muscles via the spinal cord. This sensorimotor common output path for both motor and mirroringbased representations makes primary motor and primary somatosensory areas strategic for inhibitory control and monitoring mechanisms.

\section{Physiological perspective Mu rhythms}

Although no mirror-type neurons (except see [79]) have been reported in sensorimotor cortex, of particular importance is that studies using electroencephalography and magnetoencephalography have indicated that power in mu rhythm oscillations in this region, including alpha (8$13 \mathrm{~Hz}$ ) and beta (14-25 Hz) components, is modulated by the observation and imagination of movement in the same way that self movement produces such modulation $[100,101]$. It has been known since the early 1950s that planning and execution of movement, especially of the hand, produces desynchronization or suppression of this rhythm [102,103], while inhibition of motor behavior enhances it or produces synchronization in animals [104]. This has led to a taxonomy of mu rhythm proper- ties [103]. Hari et al. [13] were the first to show an involvement of primary motor cortex in the human mirror neuron system by showing modulation of the beta (20 $\mathrm{Hz}$ ) component of the mu rhythm during the observation of hand actions. They have provided extensive magnetoencephalography evidence that primary motor cortex is activated both during the observation and execution of motor tasks $[105,106]$.

\section{Mu rhythm properties}

Using high-density, whole-head magnetoencephalography recordings and surface Laplacian transformations, a number of studies have shown that the alpha and beta mu oscillations have their origin in sensorimotor cortex [107]. However, the sources of the beta component appear to be more anterior to those of the alpha component, which originate in postcentral somatosensory cortex [108]. Indeed, significant negative correlations between both $10-\mathrm{Hz}$ and $20-\mathrm{Hz}$ mu rhythms and blood-oxygen level dependent signals have been reported in frontal and parietal cortices [109,110]. Caetano et al. [107] indicated that the modulation of the alpha rhythm lasted approximately $600 \mathrm{~ms}$ longer during action versus observation or listening conditions. They attribute this to a proprioceptive feedback signal during self movement and proposed that such a signal may enable the mirroring system to attribute agency to the correct source. It is also the case that the difference in coding action in distinct coordinate systems proposed by Kilner and Frith [43] maps well onto the alpha and beta components of mu oscillation.

Furthermore, recent studies have shown that synchronized mu rhythms in the hand area of motor cortex produces desynchronized mu rhythms in the foot or tongue area $[101,111]$ suggesting a lateral inhibitory network in sensorimotor regions. Furthermore, the differential reactivity of the mu oscillations to different contingencies suggests the existence of distinct bands: one that is somatotopically non-specific $(8-10 \mathrm{~Hz})$, one that is somatotopically specific (10-13 Hz) [112], and one (14$30 \mathrm{~Hz}$ ) that may reflect corticomuscular processes [113,114].

\section{Relationship to mirroring}

Until recently, mirror neurons had not been directly reported in sensorimotor cortex creating a problem relating the changes in mu rhythms to activity in the mirror neuron system. One explanation for the functional similarities was that sensorimotor activity involved a downstream modulation, via cortico-cortical connections, from premotor areas, including inferior frontal gyrus [12]. As was argued previously, the inferior frontal gyrus and sensorimotor cortex are reciprocally interconnected. However, this raises a potential problem. If sensorimotor cortex is activated by premotor commands during the 
observation of actions, which are similar to the motor commands generated during the behavior itself, then how is it possible to differentiate between the two and avoid movement when we observe actions? The most intuitive explanation is that the motor activity we observe is being actively gated by upstream and downstream areas. Indeed, we would argue that changes in mu rhythm reflect such signal gating. Hummel et al. [115] have shown that a significant increase in 11-13 Hz oscillations over sensorimotor cortex occurs during inhibitory control of a memory trace, while during retrieval of the trace there was a decrease in such oscillations. Results from other human electroencephalographic studies suggest that an increase in power in the beta range is associated with inhibition of the excitatory state of the motor cortex [116]. There is also clinical evidence regarding the origin of this inhibition in patients with frontal lobe damage that exhibit 'unwilled' automatic movements [117]. These clinical studies suggest that the prefrontal, anterior cingulate, and supplementary motor cortices may contribute the necessary inhibition to prevent triggering of movement commands realized in activated motor and premotor cortical areas.

Measuring cortico-spinal excitability by using transcranial magnetic stimulation during action observation has proven to be an excellent way to explore how neural networks are involved in the mirror neuron system and hence in social cognition. These studies have shown that the observation of action affects motor corticospinal $[36,118]$, intracortical [119], or spinal excitability [120]. Furthermore, such stimulation appears to desynchronize rhythms in the primary motor cortex $[13,60,121]$ strongly suggesting that mirror neurons from ventral premotor cortex modulate activity in primary motor cortex.

\section{Functional perspective}

Numerous electroencephalography, magnetoencephalography, and transcranial magnetic stimulation studies have shown that changes in mu rhythm oscillations during both execution and observation of actions reflects mirroring properties. Mu suppression has been observed during the observation of moving hands compared to the observation of bouncing balls [30], point-light biological movements [32,122], complex social interactions [31], and familiar versus unfamiliar actions [33] indicating that mu rhythms in humans are not only sensitive to objectdirected movement but to general biological motion having social significance. Mu rhythm suppression is typically greater during the execution of object-directed hand movement compared to simple hand movement. Likewise, it is greater during object-directed hand movement observation than in simple hand position observation $[123,124]$. These phenomenological properties resemble what has been reported for monkey mirror neurons. Both respond to execution and observation of object-directed movement [23], as well as cognitive imagery. Their overlapping neural sources in sensorimotor frontoparietal networks further support the argument that they are related and involved in linking perception to action, which may be a critical component in the development of higher level cognition.

Although mirror neurons are primarily thought to be involved in perception and understanding of motor actions [4], they may also play a critical role in higher order cognitive processes such as imitation $[44,50,125]$, theory of mind $[7,47,126]$, language $[50,127,128]$ and empathy [129]. A number of studies performed over the past several decades suggest that children and adults with autism spectrum disorder suffer from impairments that closely parallel the functioning of the mirror neuron system [130-133]. Indeed, the DSM-IV diagnostic criteria for autism spectrum disorders include deficits in social and communicative skills such as imitation, empathy, and shared attention, as well as restricted interests and repetitive patterns of behaviors. Elucidating their neuroetiology has been a challenge because behavioral manifestations vary both in severity as well as expression, such as Autism (low-, medium, high-functioning), Asperger's Disorder, or pervasive developmental disorder - not otherwise specified or PDD-NOS $[134,135]$. To date, no single explanation has been able to account for the broad and varied profile of these deficits [136]. However, a recent convergence of evidence on autism spectrum disorders has implicated the mirror neuron system. In fact, Williams et al. $[137,138]$ suggested that early failures of this system could result in the cascade of developmental impairments seen in autism.

Though recognized over 50 years ago, the cause of imitation impairments in autism has yet to be identified, but several hypotheses about its origin have been proposed. One hypothesis suggests that this is a core deficit that could impede early affective, social and communicative development [139]. Specifically, it is suggested that imitation deficits result from an inability to form and coordinate social representations of self and others via amodal or cross-modal representation processes - the type of function ascribed to mirror neurons. Neuroimaging and neurophysiological studies support this argument $[30,133,140]$. However, the hypothesis has been challenged recently, especially the existence of mirroringbased imitation deficits [141,142].

Nonetheless, the discovery of mirror neurons provides a testable basis for some of the major deficits seen in autism spectrum disorders. These specialized cells show increased firing rates not only during execution of an action (motor representation) but also during 'observation' of the corresponding action performed by others (visual representa- 
tion) [4]. The mirror neuron system thus appears capable of directly mimicking the action it perceives, or performing a simulation of the action without accompanying motor execution. This type of observation/execution matching system is hypothesized to provide a mechanism for translating seeing into doing, an ability that may be especially critical for imitation learning but also for the development of empathy, and theory of mind. Therefore, a number of strands of convergent evidence provide the rationale for a non-invasive investigation of the mirror neuron system in autism and for studying the effects of an intervention strategy centered on mirroring function. First, there is relatively direct evidence for mirror neuron system involvement in autism spectrum disorders. Second, many known impairments affect functional domains potentially associated with the mirror neuron system, such as imitation and theory of mind [137]. Third, there is increasing evidence for an electrophysiological signature of mirroring activity. Finally, activity-dependent reorganization is a neural property that can be effectively recruited for the remediation of disordered behavior.

\section{Conclusion}

Theories of knowledge representation can be categorized by whether or not they resort to 'embodied' versus 'disembodied' explanations [143]. Embodied theories argue that conceptual content and sensorimotor content are essentially the same, whereas disembodied theories see sensorimotor explanations as necessary but not sufficient to explain action concepts [62]. Embodied theories, therefore, argue for a central role of sensorimotor transformations in the representation of conceptual knowledge and assume that simulation requires a reactivation of sensorimotor areas. These ideas have been put forth as motor theories of action recognition, suggesting that motor processes are involved in the recognition of visually presented actions [144]. Furthermore, it has been suggested that sensorimotor processes characterize the "...semantic content of concepts in terms of the way we function without bodies in the world" and thus are intimately involved in language, theory of mind, and conceptual processing [126].

The arguments we have made in this paper, based on anatomical, physiological, modeling, and functional perspectives, are consistent with embodied explanations. That is, sensorimotor transformations are a critical component of an extended mirroring system and necessary not only for computing the patterns of muscle activation and kinematics during action observation but for simulation and understanding. Furthermore, sensorimotor transformations and the anatomical connections of sensorimotor cortex with core and extended mirror neuron system areas provide potential answers to the development, correspondence and control problems in mirroring. Nishitani and Hari [60] have shown with magnetoencephalography that activity in primary motor cortex during action observation occurs later than inferior frontal gyrus. This suggests that sensorimotor contributions to the understanding of the actions of others may be at the output end of mirror neuron system processing. As a final output path for motor and simulation-based representations, sensorimotor cortex allows for what is perhaps the critical property of mirroring systems - evoking motor representations through the observation of movement. Thus, sensorimotor cortex offers a solution to some of the more serious problems posed by mirroring systems because it offers a common output path for motor control and simulation-based transformations. These transformations can also become the foundational cornerstone for higher order social processes, such as motor learning, action understanding, imitation, perspective taking, understanding facial emotions, and empathy $[4,5]$. Furthermore, they help connect the neurophysiology of mu rhythms to the process of mirroring.

Until recently, the sensorimotor cortex has not been considered part of a mirroring system primarily because no evidence existed that neurons in these regions responded to the passive observation of actions. However, a number of studies reviewed above $[13,79,145]$ have provided support for the idea that 'mirror-like' properties occur in sensorimotor neurons to the observation of actions, including changes in mean firing rate, sensitivity to preferred direction and to the presence of a target, as well as oscillatory power modulation in specific frequency bands, raising the prospect that these areas are indeed an integral and necessary part of an extended mirroring system.

Sensorimotor learning, presumably mediated through Hebbian synaptic plasticity and auto-associational mechanisms, appears to answer the questions regarding the development of the mirror neuron system. This clearly suggests that the mirror neuron system is neither entirely innate nor inflexible and in fact may dynamically adjust to changing inputs. This gives some basis to the notion that dysfunctional mirroring systems, such as have been reported in children and adults with autism spectrum disorders, may be susceptible to therapeutic improvement with the right type of input $[33,146]$. Our own conceptual model of how mirroring develops has been particularly influenced by the work of Kilner et al. [96], which can be described as a probabilistic matching mechanism. These authors argue that one problem in inferring the cause or an intention of an action is that the problem is ill-posed "because identical movements can be made when performing different actions with different goals." The mirror neuron system and other such systems solve this problem, it is argued, by the use of predictive coding on the basis of Bayesian inference. This means that the likely cause of an observed action is inferred by minimizing the prediction 
error at all levels of the hierarchy involved during actionobservation. This type of model assumes that the areas involved in action understanding are arranged hierarchically and that the connections between them are reciprocal. The developmental time course of such wiring quite likely determines the types of mirroring processes that come online, from mimicry to functional context-sensitivities during action observation.

Solutions to the correspondence problem have required the existence of general representations of the body that are shared between observer and observed agent. The discovery of mirroring systems is consistent with that solution. That is, automatic activation of existing motor representations in sensorimotor cortex constrains the body representation mapping that occurs between observer and observed agents even when these agents do not share the same embodiment and affordances, i.e., all "action possibilities" latent in the environment [17]. This means that the system takes advantage of internal rather than external observation and thus imitation or learning occur from actions made by oneself or made by another on oneself [147].

Finally, the solution to the control problem in mirroring is grounded in the final common path architecture of sensorimotor cortex for both motor and simulation-based representations. This allows for shared access to inhibitory control circuits. To that end, changes in oscillatory activity in the mu band appear to reflect such control. Thus, the weight of the evidence suggests that sensorimotor circuits are part and parcel of the two hierarchically-organized, overlapping and interacting neural systems that have evolved and developed to manage self-other distinctions and hence social interactions [1].

\section{Competing interests}

The author declares that they have no competing interests.

\section{Acknowledgements}

I would like to thank Richard Lewis, Emma Marxer-Tobler, Derrick Asher, Jia-Min Bai, Matthew Schalles, Albert Ayala, Aaron Cortez, Nick Pojman, Alicia Trigerio, and Oriana Clark for early help with the paper. Oriana Clark was also instrumental in the design of the figures.

\section{References}

I. Grafton ST, Hamilton AF: Evidence for a distributed hierarchy of action representation in the brain. Hum Mov Sci 2007, 26:590-616.

2. Bernstein NA: On dexterity and its development. In Dexterity and its Development Edited by: Latash ML, Turvey MT. Mahwah, New Jersey: Lawrence Erlbaum Associates; 1996.

3. Hurley S: The shared circuits model (SCM): how control, mirroring, and simulation can enable imitation, deliberation, and mindreading. Behav Brain Sci 2008, 3 I : I-22

4. Rizzolatti G, Craighero L: The mirror-neuron system. Annu Rev Neurosci 2004, 27:169-192.

5. Lyons DE, Santos LR, Keil FC: Reflections of other minds: how primate social cognition can inform the function of mirror neurons. Curr Opin Neurobiol 2006, I 6:230-234.
6. Decety J, Jackson PL: The functional architecture of human empathy. Behav Cogn Neurosci Rev 2004, 3:7I-I00.

7. Gallese V: The roots of empathy: The shared manifold hypothesis and the neural basis of intersubjectivity. Psychopathology 2003, 36:171-180.

8. Keysers C, Gazzola V: Towards a unifying neural theory of social cognition. Prog Brain Res 2006, I 56:379-40I.

9. Meltzoff AN, Decety J: What imitation tells us about social cognition: a rapprochement between developmental psychology and cognitive neuroscience. Philos Trans R Soc Lond B Biol Sci 2003, 358:49l-500.

10. Falck-Ytter T, Gredeback G, von HC: Infants predict other people's action goals. Nat Neurosci 2006, 9:878-879.

II. Ferrari PF, Visalberghi E, Paukner A, Fogassi L, Ruggiero A, Suomi SJ: Neonatal imitation in rhesus macaques. PLoS Biol 2006, 4:e302.

12. Pineda JA: The functional significance of mu rhythms: translating "seeing" and "hearing" into "doing". Brain Res Brain Res Rev 2005, 50:57-68.

13. Hari R, Forss N, Avikainen S, Kirveskari E, Salenius S, Rizzolatti G: Activation of human primary motor cortex during action observation: a neuromagnetic study. Proc Natl Acad Sci USA 1998, 95:|506|-|5065.

14. Shimada S, Hiraki K: Infant's brain responses to live and televised action. Neuroimage 2006, 32:930-939.

I5. Lepage JF, Theoret H: The mirror neuron system: grasping others' actions from birth? Dev Sci 2007, 10:5 I3-523.

16. Lepage JF, Theoret H: EEG evidence for the presence of an action observation-execution matching system in children. Eur J Neurosci 2006, 23:2505-2510.

17. Brass M, Heyes C: Imitation: is cognitive neuroscience solving the correspondence problem? Trends Cogn Sci 2005, 9:489-495.

18. Brass M, Derrfuss J, Matthes-von CG, von Cramon DY: Imitative response tendencies in patients with frontal brain lesions. Neuropsychology 2003, I 7:265-27I.

19. Leslie KR, Johnson-Frey SH, Grafton ST: Functional imaging of face and hand imitation: towards a motor theory of empathy. Neuroimage 2004, 21:601-607.

20. Muthukumaraswamy SD, Singh KD: Modulation of the human mirror neuron system during cognitive activity. Psychophysiology 2008.

21. Arbib M: Autism - more than the mirror system. Clinical Neuropsychiatry 2007, 4:208-222.

22. Rizzolatti G, Gallese V: Do perception and action result from different brain circuits? The three visual systems hypothesis. In 23 problems in systems neuroscience Edited by: Van Hemmen JL, Sejnowski TJ. New York: Oxford University Press; 2006:369-393.

23. di Pellegrino G, Fadiga L, Fogassi L, Gallese V, Rizzolatti G: Understanding motor events: a neurophysiological study. Exp Brain Res 1992, 91: |76-180.

24. Gallese V, Fadiga L, Fogassi L, Rizzolatti G: Action recognition in the premotor cortex. Brain 1996, I I 9(Pt 2):593-609.

25. Kohler E, Keysers C, Umilta MA, Fogassi L, Gallese V, Rizzolatti G: Hearing sounds, understanding actions: action representation in mirror neurons. Science 2002, 297:846-848.

26. Keysers C, Kohler E, Umilta MA, Nanetti L, Fogassi L, Gallese V: Audiovisual mirror neurons and action recognition. Exp Brain Res 2003, I 53:628-636.

27. Mukamel R, Ekstrom AD, Kaplan JT, lacoboni M, Fried I: Mirror properties of single cells in human medial frontal cortex [abstract]. Society for Neuroscience Abstracts 2007, 127 :.

28. Binkofski $F$, Buccino $G$ : The role of ventral premotor cortex in action execution and action understanding. J Physiol Paris 2006, 99:396-405.

29. Binkofski F, Buccino G: Motor functions of the Broca's region. Brain Lang 2004, 89:362-369.

30. Oberman LM, Hubbard EM, McCleery JP, Altschuler EL, Ramachandran VS, Pineda JA: EEG Evidence for Mirror Neuron Dysfunction in Autism Spectrum Disorders. Cognitive Brain Research 2005.

31. Oberman LM, Pineda JA, Ramachandran VS: The Human Mirror Neuron System: A Link Between Action Observation and Social Skills. Soc Cog Affect Neurosci 2006.

32. Ulloa ER, Pineda JA: Recognition of point-light biological motion: mu rhythms and mirror neuron activity. Behav Brain Res 2007, 183:188-194. 
33. Oberman LM, Ramachandran VS, Pineda JA: Modulation of mu suppression in children with autism spectrum disorders in response to familiar or unfamiliar stimuli: The mirror neuron hypothesis. Neuropsychologia 2008.

34. Hauk O, Pulvermuller F: Neurophysiological distinction of action words in the fronto-central cortex. Hum Brain Mapp 2004, 2 I: | $91-201$.

35. Kilner JM, Marchant JL, Frith CD: Modulation of the mirror system by social relevance. Soc Cogn Affect Neurosci 2006, I : | 43- | 48.

36. Fadiga L, Buccino G, Craighero L, Fogassi L, Gallese V, Pavesi G: Corticospinal excitability is specifically modulated by motor imagery: a magnetic stimulation study. Neuropsychologia 1999, 37:| $47-158$

37. Chaminade T, Meary D, Orliaguet JP, Decety J: Is perceptual anticipation a motor simulation? A PET study. Neuroreport 200I, I 2:3669-3674.

38. Chaminade T, Meltzoff AN, Decety J: Does the end justify the means? A PET exploration of the mechanisms involved in human imitation. Neuroimage 2002, I5:318-328.

39. lacoboni M, Woods RP, Brass M, Bekkering H, Mazziotta JC, Rizzolatti G: Cortical mechanisms of human imitation. Science 1999 286:2526-2528.

40. Buccino G, Binkofski F, Fink GR, Fadiga L, Fogassi L, Gallese V, Seitz RJ, Zilles K, Rizzolatti G, Freund HJ: Action observation activates premotor and parietal areas in a somatotopic manner: an fMRI study. Eur J Neurosci 200I, I 3:400-404.

4I. Buccino $G$, Binkofski F, Riggio L: The mirror neuron system and action recognition. Brain Lang 2004, 89:370-376.

42. Jellema T, Perrett DI: Cells in monkey STS responsive to articulated body motions and consequent static posture: a case of implied motion? Neuropsychologia 2003, 41: I728-I737.

43. Puce A, Perrett D: Electrophysiology and brain imaging of biological motion. Philos Trans R Soc Lond B Biol Sci 2003, 358:435-445.

44. Rizzolatti G, Fogassi L, Gallese V: Neurophysiological mechanisms underlying the understanding and imitation of action. Nat Rev Neurosci 200I, 2:66I-670.

45. Umilta MA, Kohler E, Gallese V, Fogassi L, Fadiga L, Keysers C, Rizzolatti G: I know what you are doing. a neurophysiological study. Neuron 200I, 3 I:I55-165.

46. Blakemore SJ, Frith CD, Wolpert DM: The cerebellum is involved in predicting the sensory consequences of action. Neuroreport 200।, I 2: |879-1884

47. Gallese V, Keysers C, Rizzolatti G: A unifying view of the basis of social cognition. Trends Cogn Sci 2004, 8:396-403.

48. Keysers C, Gazzola V: Unifying social cognition. In The Role of Mirroring Processes in Social Cognition Edited by: Pineda JA. San Diego, CA: Humana Press; 2008.

49. Oztop E, Arbib MA: Schema design and implementation of the grasp-related mirror neuron system. Biol Cybern 2002, 87: I 16-140.

50. Oztop $E$, Kawato $M$, Arbib M: Mirror neurons and imitation: computationally guided review. Neural Netw 2006, 19:254-27I.

5I. Kilner JM, Frith CD: Action observation: inferring intentions without mirror neurons. Curr Biol 2008, I 8:R32-R33.

52. Jellema T, Perrett DI: Neural basis for the perception of goaldirected actions. In The cognitive neuroscience of social behavio Edited by: Easton A, Emery NJ. Hove/New York: Psychology Press; 2005:8I-II2.

53. Damasio A: Mental self: The person within. Nature 2003 , 423:227.

54. Singer T, Seymour B, O'Doherty J, Kaube H, Dolan RJ, Frith CD: Empathy for pain involves the affective but not sensory components of pain. Science 2004, 303: I I57-I 162

55. Saarela MV, Hlushchuk Y, Williams AC, Schurmann M, Kalso E, Har $\mathrm{R}$ : The compassionate brain: humans detect intensity of pain from another's face. Cereb Cortex 2007, I 7:230-237.

56. Cisek P, Kalaska JF: Neural correlates of mental rehearsal in dorsal premotor cortex. Nature 2004, 43 I:993-996.

57. Filimon F, Nelson JD, Hagler DJ, Sereno MI: Human cortical representations for reaching: mirror neurons for execution observation, and imagery. Neuroimage 2007, 37:1315-1328.

58. Grezes J, Armony JL, Rowe J, Passingham RE: Activations related to "mirror" and "canonical" neurones in the human brain: an fMRI study. Neuroimage 2003, I 8:928-937.
59. Gazzola V, ziz-Zadeh L, Keysers C: Empathy and the somatotopic auditory mirror system in humans. Curr Biol 2006, 16:1824-1829.

60. Nishitani N, Avikainen S, Hari R: Abnormal imitation-related cortical activation sequences in Asperger's syndrome. Ann Neurol 2004, 55:558-562.

61. Keysers C, Wicker B, Gazzola V, Anton JL, Fogassi L, Gallese V: A touching sight: SII/PV activation during the observation and experience of touch. Neuron 2004, 42:335-346.

62. Barsalou LW, Kyle SW, Barbey AK, Wilson CD: Grounding conceptual knowledge in modality-specific systems. Trends Cogn Sci 2003, 7:84-91.

63. Barsalou LW: Grounded cognition. Annu Rev Psychol 2008, 59:617-645

64. Niedenthal PM, Barsalou LW, Winkielman P, Krauth-Gruber S, Ric F: Embodiment in attitudes, social perception, and emotion. Pers Soc Psychol Rev 2005, 9: I84-2। I.

65. Sommerville JA, Decety J: Weaving the fabric of social interaction: articulating developmental psychology and cognitive neuroscience in the domain of motor cognition. Psychon Bull Rev 2006, 13:179-200.

66. Adolphs R: How do we know the minds of others? Domainspecificity, simulation, and enactive social cognition. Brain Res 2006, 1079:25-35.

67. Jackson PL, Meltzoff AN, Decety J: How do we perceive the pain of others? A window into the neural processes involved in empathy. Neuroimage 2005, 24:771-779.

68. Fadiga L, Craighero L: Electrophysiology of action representation. I Clin Neurophysiol 2004, 2 I: I 57-169.

69. Cochin S, Barthelemy C, Roux S, Martineau J: Observation and execution of movement: similarities demonstrated by quantified electroencephalography. Eur J Neurosci 1999, I I: I839-1842.

70. Altschuler EL, Vankov A, Wang V, Ramachandran VS, Pineda JA: Person see, person do: Human cortical electrophysiological correlates of monkey see monkey do cells. Soc Neurosci Abst 1997.

7I. Grafton ST, Fagg AH, Woods RP, Arbib MA: Functional anatomy of pointing and grasping in humans. Cereb Cortex 1996, 6:226-237.

72. Decety J, Grezes J: The power of simulation: imagining one's own and other's behavior. Brain Res 2006, 1079:4-I4.

73. lacoboni M: Neural mechanisms of imitation. Curr Opin Neurobiol 2005, I 5:632-637.

74. Stepniewska I, Preuss TM, Kaas JH: Architectonics, somatotopic organization, and ipsilateral cortical connections of the primary motor area (MI) of owl monkeys. J Comp Neurol I993, 330:238-27I.

75. Stepniewska I, Preuss TM, Kaas JH: Ipsilateral cortical connections of dorsal and ventral premotor areas in New World owl monkeys. J Comp Neurol 2006, 495:691-708.

76. Greenlee JD, Oya H, Kawasaki H, Volkov IO, Kaufman OP, Kovach C, Howard MA, Brugge JF: A functional connection between inferior frontal gyrus and orofacial motor cortex in human. J Neurophysiol 2004, 92: I I53- I I64

77. Dum RP, Strick PL: Frontal lobe inputs to the digit representations of the motor areas on the lateral surface of the hemisphere. J Neurosci 2005, 25:1375-1386.

78. He SQ, Dum RP, Strick PL: Topographic organization of corticospinal projections from the frontal lobe: motor areas on the lateral surface of the hemisphere. J Neurosci 1993, I 3:952-980.

79. Tkach D, Reimer J, Hatsopoulos NG: Congruent activity during action and action observation in motor cortex. J Neurosci 2007, 27: | 324|- I3250.

80. Stefan K, Cohen LG, Duque J, Mazzocchio R, Celnik P, Sawaki L, Ungerleider L, Classen J: Formation of a motor memory by action observation. I Neurosci 2005, 25:9339-9346.

81. Keysers C, Wicker B, Gazzola V, Anton JL, Fogassi L, Gallese V: A touching sight: SII/PV activation during the observation and experience of touch. Neuron 2004, 42:335-346.

82. Cipolloni PB, Pandya DN: Cortical connections of the frontoparietal opercular areas in the rhesus monkey. J Comp Neurol 1999, 403:431-458.

83. Blakemore SJ, Frith C: The role of motor contagion in the prediction of action. Neuropsychologia 2005, 43:260-267. 
84. Damasio AR: The somatic marker hypothesis and the possible functions of the prefrontal cortex. Philos Trans $R$ Soc Lond B Biol Sci 1996, 35 I: |413-1420.

85. Adolphs R, Damasio H, Tranel D, Cooper G, Damasio AR: A role for somatosensory cortices in the visual recognition of emotion as revealed by three-dimensional lesion mapping. J Neurosci 2000, 20:2683-2690.

86. Hagen MC, Zald DH, Thornton TA, Pardo JV: Somatosensory processing in the human inferior prefrontal cortex. I Neurophysiol 2002, 88: |400-|406.

87. Romo R, Hernandez A, Zainos A, Brody C, Salinas E: Exploring the cortical evidence of a sensory-discrimination process. Philos Trans R Soc Lond B Biol Sci 2002, 357:1039-105I.

88. Catmur $C$, Walsh V, Heyes C: Sensorimotor learning configures the human mirror system. Curr Biol 2007, I 7: | 527-I53 I.

89. Heyes C: Causes and consequences of imitation. Trends Cogn Sci 200I, 5:253-26I.

90. Keysers C, Perrett DI: Demystifying social cognition: a Hebbian perspective. Trends Cogn Sci 2004, 8:501-507.

91. Calvo-Merino B, Glaser DE, Grezes J, Passingham RE, Haggard P Action observation and acquired motor skills: an FMRI study with expert dancers. Cereb Cortex 2005, I 5: I 243- I 249.

92. Haslinger B, Erhard P, Altenmuller E, Hennenlotter A, Schwaiger M, Grafin von EH, Rummeny E, Conrad B, Ceballos-Baumann AO Reduced recruitment of motor association areas during bimanual coordination in concert pianists. Hum Brain Mapp 2004, 22:206-2I5.

93. Fagg $\mathrm{AH}$, Arbib MA: Modeling parietal-premotor interactions in primate control of grasping. Neural Netw I 998, I I: | 277-I303.

94. Todorov E: Optimality principles in sensorimotor control. Nat Neurosci 2004, 7:907-915.

95. Todorov E, Jordan MI: Optimal feedback control as a theory of motor coordination. Nat Neurosci 2002, 5: I 226-I 235.

96. Kilner JM, Friston KJ, Frith CD: The mirror-neuron system: a Bayesian perspective. Neuroreport 2007, 18:619-623.

97. Kakei S, Hoffman DS, Strick PL: Sensorimotor transformations in cortical motor areas. Neurosci Res 2003, 46: I- I0.

98. Kakei S, Hoffman DS, Strick PL: Direction of action is represented in the ventral premotor cortex. Nat Neurosci 2001, 4: $1020-1025$

99. Kakei S, Hoffman DS, Strick PL: Muscle and movement representations in the primary motor cortex. Science 1999, 285:2। 36-2139.

100. Pineda JA, Allison BZ, Vankov A: The effects of self-movement, observation, and imagination on $\mathrm{mu}$ rhythms and readiness potentials (RP's): toward a brain-computer interface (BCI). IEEE Trans Rehabil Eng 2000, 8:219-222.

101. Neuper C, Wortz M, Pfurtscheller G: ERD/ERS patterns reflecting sensorimotor activation and deactivation. Prog Brain Res 2006, I 59:2 I |-222.

102. Gastaut HJ, Bert J: EEG changes during cinematographic presentation. Electroencephalogr Clin Neurophysiol 1954, 6:433-444.

103. Kuhlman WN: Functional topography of the human mu rhythm. Electroencephalogr Clin Neurophysiol 1978, 44:83-93.

104. Howe RC, Sterman MB: Cortical-subcortical EEG correlates of suppressed motor behavior during sleep and waking in the cat. Electroencephalogr Clin Neurophysiol 1972, 32:681-695.

105. Hari R: Action-perception connection and the cortical mu rhythm. Prog Brain Res 2006, I 59:253-260.

106. Hari R, Salmelin R, Makela JP, Salenius S, Helle M: Magnetoencephalographic cortical rhythms. Int J Psychophysiol 1997, 26:51-62.

107. Caetano G, Jousmaki V, Hari R: Actor's and observer's primary motor cortices stabilize similarly after seen or heard motor actions. Proc Natl Acad Sci USA 2007, 104:9058-9062.

108. Salmelin R, Hari R: Characterization of spontaneous MEG rhythms in healthy adults. Electroencephalogr Clin Neurophysio 1994, 9 1:237-248.

109. Laufs H, Kleinschmidt A, Beyerle A, Eger E, Salek-Haddadi A, Preibisch C, Krakow K: EEG-correlated fMRI of human alpha activity. Neuroimage 2003, 19:|463-1476.

I 10. Laufs H, Krakow K, Sterzer P, Eger E, Beyerle A, Salek-Haddadi A Kleinschmidt A: Electroencephalographic signatures of attentional and cognitive default modes in spontaneous brain activity fluctuations at rest. Proc Natl Acad Sci USA 2003, 100: I 1053-। 1058.
III. Pfurtscheller G, Neuper C, Andrew C, Edlinger G: Foot and hand area mu rhythms. Int J Psychophysiol 1997, 26: I2 I- I35.

I I2. Pfurtscheller G, Neuper C, Krausz G: Functional dissociation of lower and upper frequency mu rhythms in relation to voluntary limb movement. Clin Neurophysiol 2000, I I I: I873- I879.

I I3. Hari R, Salmelin R: Human cortical oscillations: a neuromagnetic view through the skull. Trends Neurosci 1997, 20:44-49.

I 14. Salenius S, Hari R: Synchronous cortical oscillatory activity during motor action. Curr Opin Neurobiol 2003, I3:678-684.

I I5. Hummel F, Andres F, Altenmuller E, Dichgans J, Gerloff C: Inhibitory control of acquired motor programmes in the human brain. Brain 2002, 1 25:404-420.

I 16. Gilbertson T, Lalo E, Doyle L, Di LV, Cioni B, Brown P: Existing motor state is favored at the expense of new movement during I3-35 Hz oscillatory synchrony in the human corticospinal system. J Neurosci 2005, 25:777I-7779.

I 17. Archibald SJ, Mateer CA, Kerns KA: Utilization behavior: clinical manifestations and neurological mechanisms. Neuropsychol Rev 2001, I I:I 17-130.

I I8. Maeda F, Kleiner-Fisman G, Pascual-Leone A: Motor facilitation while observing hand actions: specificity of the effect and role of observer's orientation. J Neurophysiol 2002, 87: $1329-1335$.

I19. Strafella AP, Paus T: Modulation of cortical excitability during action observation: a transcranial magnetic stimulation study. Neuroreport 2000, I I:2289-2292

120. Baldissera F, Cavallari P, Craighero L, Fadiga L: Modulation of spinal excitability during observation of hand actions in humans. Eur J Neurosci 2001, I 3:190-194.

I2I. Nishitani N, Hari R: Temporal dynamics of cortical representation for action. Proc Natl Acad Sci USA 2000, 97:913-918.

122. Kessler K, Biermann-Ruben K, Jonas M, Siebner HR, Baumer T, Munchau $A$, Schnitzler A: Investigating the human mirror neuron system by means of cortical synchronization during the imitation of biological movements. Neuroimage 2006, 33:227-238.

123. Muthukumaraswamy SD, Johnson BW, McNair NA: Mu rhythm modulation during observation of an object-directed grasp. Brain Res Cogn Brain Res 2004, 19:195-201.

124. Muthukumaraswamy SD, Johnson BW: Changes in rolandic mu rhythm during observation of a precision grip. Psychophysiology 2004, 41 : I52-156.

125. Arbib MA, Billard A, lacoboni M, Oztop E: Synthetic brain imaging: grasping, mirror neurons and imitation. Neural Netw 2000 , I 3:975-997.

126. Gallese V: Before and below 'theory of mind': embodied simulation and the neural correlates of social cognition. Philos Trans R Soc Lond B Biol Sci 2007.

127. Arbib MA, Billard A, lacoboni M, Oztop E: Synthetic brain imaging: grasping, mirror neurons and imitation. Neural Netw 2000, 13:975-997.

128. Rizzolatti G, Arbib MA: Language within our grasp. Trends Neurosci 1998, 2 I: 188-194.

129. Carr L, lacoboni M, Dubeau MC, Mazziotta JC, Lenzi GL: Neura mechanisms of empathy in humans: a relay from neural systems for imitation to limbic areas. Proc Natl Acad Sci USA 2003, 100:5497-5502.

130. Receveur C, Lenoir P, Desombre H, Roux S, Barthelemy C, Malvy J: Interaction and imitation deficits from infancy to 4 years of age in children with autism: a pilot study based on videotapes. Autism 2005, 9:69-82

|3I. Rogers SJ, Hepburn SL, Stackhouse T, Wehner E: Imitation performance in toddlers with autism and those with other developmental disorders. J Child Psychol Psychiatry 2003, 44:763-781.

132. Williams JH, Whiten A, Singh T: A systematic review of action imitation in autistic spectrum disorder. I Autism Dev Disord 2004, 34:285-299.

133. Bernier R, Dawson G, Webb S, Murias M: EEG mu rhythm and imitation impairments in individuals with autism spectrum disorder. Brain Cogn 2007, 64:228-237.

134. Matson JL: Current status of differential diagnosis for children with autism spectrum disorders. Res Dev Disabil 2007 28(2): $109-1 \mid 8$

135. Volkmar FR, Klin A, Siegel B, Szatmari P, Lord C, Campbell M, Freeman BJ, Cicchetti DV, Rutter M, Kline W: Field trial for autistic disorder in DSM-IV. Am J Psychiatry 1994, I 5 I:| |36 |-1367. 
136. Muller RA: The study of autism as a distributed disorder. Ment Retard Dev Disabil Res Rev 2007, 13:85-95.

137. Williams JH, Whiten A, Suddendorf T, Perrett DI: Imitation, mirror neurons and autism. Neurosci Biobehav Rev 200I, 25:287-295.

138. Williams JH, Waiter GD, Gilchrist A, Perrett DI, Murray AD, Whiten $A$ : Neural mechanisms of imitation and 'mirror neuron' functioning in autistic spectrum disorder. Neuropsychologia 2006, 44:610-621.

139. Ozonoff S, Pennington BF, Rogers SJ: Executive function deficits in high-functioning autistic individuals: relationship to theory of mind. J Child Psychol Psychiatry 1991, 32: 108I-II05.

140. Dapretto M, Davies MS, Pfeifer JH, Scott AA, Sigman M, Bookheimer SY, lacoboni M: Understanding emotions in others: mirror neuron dysfunction in children with autism spectrum disorders. Nat Neurosci 2006, 9:28-30.

141. Leighton J, Bird G, Charman T, Heyes C: Weak imitative performance is not due to a functional 'mirroring' deficit in adults with Autism Spectrum Disorders. Neuropsychologia 2008, 46: 104I-1049.

142. Hamilton AF, Brindley RM, Frith U: Imitation and action understanding in autistic spectrum disorders: how valid is the hypothesis of a deficit in the mirror neuron system? Neuropsychologia 2007, 45: I859-I868.

143. Fadiga L, Craighero L: New insights on sensorimotor integration: from hand action to speech perception. Brain Cogn 2003, 53:5।4-524.

144. Vogt $S$, Thomaschke R: From visuo-motor interactions to imitation learning: behavioural and brain imaging studies. J Sports Sci 2007, 25:497-5I7.

145. Fecteau S, Carmant L, Tremblay C, Robert M, Bouthillier A, Theoret $\mathrm{H}$ : A motor resonance mechanism in children? Evidence from subdural electrodes in a 36-month-old child. Neuroreport 2004, 15:2625-2627.

146. Pineda JA, Moore A, Elfenbein H, Cox R: Hierarchically Organized "Mirroring" Processes in Social Cognition: The Functional Neuroanatomy of Empathy. In The Role of Mirroring Processes in Social Cognition Edited by: Pineda JA. Humana Press; 2008.

147. Saunders J, Nehaniv CL, Dautenhanh K, Alissandrakis A: Self-imitation and environmental scaffolding for robot teaching. International J Adv Robot Syst 2007, 4:109-124. http.//www.behavioralandbrainfunctions.com/content/4/1/47

\section{Publish with Biomed Central and every scientist can read your work free of charge}

"BioMed Central will be the most significant development for disseminating the results of biomedical research in our lifetime. "

Sir Paul Nurse, Cancer Research UK

Your research papers will be:

- available free of charge to the entire biomedical community

- peer reviewed and published immediately upon acceptance

- cited in PubMed and archived on PubMed Central

- yours - you keep the copyright

Submit your manuscript here:

http://www.biomedcentral.com/info/publishing_adv.asp
BioMedcentral 